\title{
Progressive Changes in Synaptic Inputs to Motoneurons in Adult Sacral Spinal Cord of a Mouse Model of Amyotrophic Lateral Sclerosis
}

\author{
Mingchen Jiang, ${ }^{1}$ Jenna E. Schuster, ${ }^{1}$ Ronggen Fu, ${ }^{2}$ Teepu Siddique, ${ }^{2}$ and C. J. Heckman ${ }^{1,3}$ \\ ${ }^{1}$ Department of Physiology, ${ }^{2}$ Davee Department of Neurology and Clinical Neurosciences, Northwestern University Feinberg School of Medicine, Chicago, \\ Illinois 60611, and ${ }^{3}$ Department of Physical Medicine and Rehabilitation, Northwestern University Feinberg School of Medicine, Chicago, Illinois 60622
}

\begin{abstract}
Amyotrophic lateral sclerosis (ALS) is characterized by progressive degeneration of motoneurons. One potential mechanism is excitotoxicity. We studied the behaviors of spinal neurons using an in vitro preparation of the sacral cord from the G93A SOD1 mouse model of ALS. Measurements were conducted at presymptomatic [approximately postnatal day $50(\sim \mathrm{P} 50)$ ], early $(\sim \mathrm{P} 90)$, and late $(>\mathrm{P} 120)$ stages of the disease. Short-latency reflexes (SRs) in ventral roots, presumably monosynaptic, were evoked by electrical stimulation of a dorsal root. The fraction of motoneurons capable of responding to this activation was evaluated by measuring the compound action potential [total motor activity (TMA)] evoked by antidromic stimulation of the distal ventral root. In mutant SOD1 (mSOD1) mice, both the SR and the TMA decreased with age compared with nontransgenic littermates, ruling out the SR as a source of increasing excitotoxicity. Spinal interneuron activity was assessed using the synchronized ventral root bursts generated by both bath application of blockers of inhibitory neurotransmitters (glycine, $\mathrm{GABA}_{\mathrm{A}}$ ) and agonists of glutamate receptors (especially NMDA receptors). After symptom onset, a higher percentage of preparations from mSOD1 mice exhibited bursting, and these bursts exhibited more sub-bursts and a more disorganized pattern. In mSOD1 mice with clear muscle tremor, the ventral roots exhibited spontaneous synchronized bursts, which were highly sensitive to the blockade of NMDA receptors. These data suggest that although short-latency sensory input does not increase as symptoms develop, interneuron activity does increase and may contribute to excitotoxicity.
\end{abstract}

\section{Introduction}

Excitotoxicity is one of several mechanisms being investigated for motoneuron degeneration in amyotrophic lateral sclerosis (ALS) (Cleveland and Rothstein, 2001; Rao and Weiss, 2004; Pasinelli and Brown, 2006). It is generally thought that excitotoxicity arises from excessive $\mathrm{Ca}^{2+}$ entry into motoneurons (Arundine and Tymianski, 2003; Wang and Zhang, 2005; Van Den Bosch et al., 2006), and is exacerbated by the poor motoneuronal $\mathrm{Ca}^{2+}$ buffering (Vanselow and Keller, 2000; von Lewinski and Keller, 2005). $\mathrm{Ca}^{2+}$ entry occurs primarily through two mechanisms: the opening of $\mathrm{Ca}^{2+}$-permeable ligand-gated channels and the activation of voltage-sensitive channels during action potential (AP) generation. Glutamatergic synaptic inputs allow $\mathrm{Ca}^{2+}$ entry through NMDA receptors (NMDARs) and AMPA receptors (AMPARs), although $\mathrm{Ca}^{2+}$ entry through AMPARs depends on the presence of the AMPA 2 subunit (Van Damme et al., 2003). $\mathrm{Ca}^{2+}$ entry also occurs during APs when voltage-sensitive $\mathrm{Na}^{+}$ channels depolarize the cell, leading to the activation of highthreshold voltage-sensitive $\mathrm{Ca}^{2+}$ channels (Powers and Binder,

Received Feb. 3, 2009; revised Sept. 14, 2009; accepted 0ct. 11, 2009

This work was supported by The ALS Association Grant 1626, National Institutes of Health Grants (NS051462 and NS050641), Les Turner ALS Foundation, and Les Turner ALS Foundation/Herbert C. Wenske Foundation Professor.

Correspondence should be addressed to Mingchen Jiang at the above address. E-mail address: m-jiang@ northwestern.edu.

D01:10.1523/JNEUROSCI.0574-09.2009

Copyright $\odot 2009$ Society for Neuroscience $\quad 0270-6474 / 09 / 2915031-08 \$ 15.00 / 0$
2001). Therefore, changes in synaptic and intrinsic motoneuron excitability may play a key role in the ALS excitotoxicity hypothesis.

Recent studies suggest that both mechanisms of $\mathrm{Ca}^{2+}$ entry are altered at a very early age in the mSOD1 mouse model (G93A) of ALS. In motoneurons cultured from embryonic mSOD1 mice, an important component of the total $\mathrm{Na}^{+}$current, the persistent $\mathrm{Na}^{+}$current (Na PIC), is markedly upregulated (Kuo et al., 2004, 2005). This phenomenon is not restricted to cultured cells, as the $\mathrm{Na}$ PIC is also clearly upregulated in neonatal brainstem motoneurons (van Zundert et al., 2008). In addition, in this mSOD1 mouse model of ALS, the Na PIC in interneurons and synaptic input to motoneurons tend to be altered in neonatal preparations (van Zundert et al., 2008). Yet, there are also other changes that may counter the effects of interneuron and motoneuron hyperexcitability, including the abnormal growth of dendrites and the reduction in input conductance seen in neonatal motoneurons in G93A and G85R mice (Durand et al., 2006; Amendola and Durand, 2008; van Zundert et al., 2008).

Studies of spinal neuronal excitability in ALS thus far have been restricted to cell culture and neonates. We have recently developed an adult in vitro preparation of the sacral spinal cord that allowed us to measure the function of spinal motor system in adult mouse (Jiang and Heckman, 2006). In this study, we focused on the changes in the short-latency sensory input and the interneuronal input to motoneurons in the adult G93A mSOD1 mouse. We tested the hypothesis that synaptic inputs, which are 
involved in modulation of motoneuron activity, are altered in mSOD1 adult mice that range in age from presymptomatic ( $\sim 50 \mathrm{~d}$ old $)$ to early $(\sim 90 \mathrm{~d})$ and late $(>120 \mathrm{~d})$ stages of the disease. Motor output via ventral roots was assessed in response to the short-latency, presumably monosynaptic input, and to the synchronized bursting generated by interneuronal circuits, as spinal interneurons are implicated in this disease (Berger et al., 1992; Nagao et al., 1998; Schütz, 2005; Stephens et al., 2006). The results indicate that the monosynaptic reflex decreases as the disease progresses, but that interneuronal excitability becomes higher than normal. These results suggest that increased excitability of spinal interneurons plays a role in the pathology of ALS, but that low-threshold short-latency sensory input does not.

\section{Materials and Methods}

Transgenic mice overexpressing the human mutant SOD1 gene (G93A, mSOD1) and their nontransgenic (NT) littermates were maintained at the Center for Comparative Medicine of Northwestern University Medical Center. All experimental procedures were reviewed and approved by the University Animal Research Committee and were in accordance with the National Institute of Health Guide for the Care and Use of Laboratory Animals. In this study, we chose to record ventral root activity in sacral segments 1-3 (S1-S3) because their reliable activity can be maintained for several hours (Jiang and Heckman, 2006).

Sacral cord preparation. For the dorsal root stimulation-evoked reflex experiments shortlatency reflexes (SRs), animals of both NT and mSOD1 mice were divided into three age groups [postnatal day 50 (P50)-P60, P90-P100, and $\mathrm{P} 120-\mathrm{P} 130]$. Experiments measuring ventral root synchronized and periodic bursting, driven by spinal interneurons (Cowley and Schmidt, 1995; Hinckley et al., 2005), were performed on mSOD1 mice older than P90. The end stage criterion for mSOD1 mice was the inability to right themselves from a supine position, similar to the standards used by Gurney's laboratory (Chiu et al., 1995). Mice meeting this criterion were not included. We selected motoneurons in the S1-S3 segments based on our previous work and more importantly because ALS-resistant motoneurons are not located in this area (Hamson et al., 2002). It is likely, as in the rat sacral cord (Bennett et al., 1999), that studied motoneurons innervate the skeletal muscle of the tail. Our behavioral studies show that flaccidity develops in the tail during the progression of the disease (J. E. Schuster and C. J. Heckman, unpublished results). The surgical procedure is described in detail in Jiang and Heckman (2006). Briefly, animals were deeply anesthetized with intraperitoneal injections of urethane $(0.18$ $\mathrm{g} / 100 \mathrm{~g}$ for all NT and P50-P60 mSOD1 mice). Significantly lower doses of urethane $(\sim 0.06-0.12 \mathrm{~g} / 100 \mathrm{~g})$ were required for mSOD1 mice P90 and older. Supplemental anesthesia was determined by the animal's response to foot pinching with forceps.

During the surgery, animals were supplied with $95 \% \mathrm{O}_{2} / 5 \% \mathrm{CO}_{2}$ through a face mask. The spinal column was opened between the low thoracic and caudal segments, and the spinal cord was immediately superfused with modified artificial CSF (mACSF) composed of the following (in mM): $20 \mathrm{NaCl}, 140$ sucrose, $3 \mathrm{KCl}, 1 \mathrm{NaH}_{2} \mathrm{PO}_{4}, 1.5 \mathrm{MgSO}_{4}, 1$ $\mathrm{CaCl}_{2}, 10$ glucose, $26 \mathrm{NaHCO}_{3}$, and 1 kynurenic acid. The osmolarity was adjusted to $340 \mathrm{mOsm}$ by adding sucrose, and the $\mathrm{pH}$ was adjusted to 7.4 by adding $\mathrm{NaHCO}_{3}$ to the $\mathrm{mACSF}$ when bubbled with $95 \% \mathrm{O}_{2} / 5 \% \mathrm{CO}_{2}$.
B
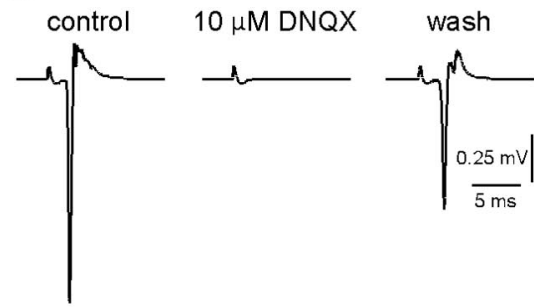

10
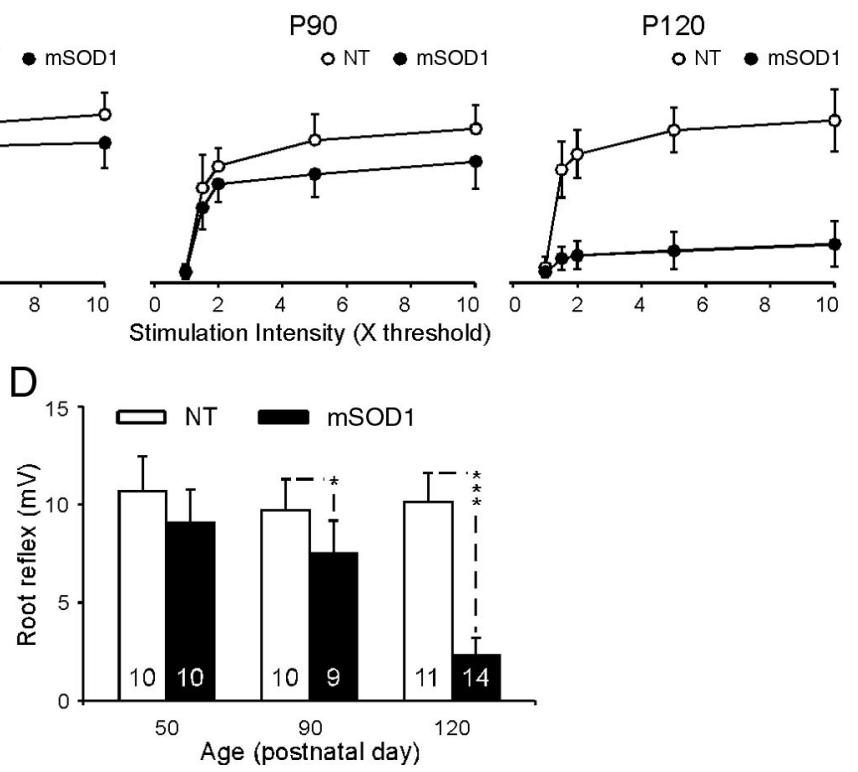

Age (postnatal day)$$
\text { (a) }
$$

Figure 1. The evoked root-reflex and its difference between NT and mSOD1 mice. $\boldsymbol{A}$, The root reflexes evoked at different stimulus intensities marked as 1-10 times threshold that just evokes a response. $\boldsymbol{B}$, Inhibition by DNQX, the antagonist for AMPA root reflexes for all three age groups. $\boldsymbol{D}$, Plot of the maximum root reflexes between NT and mSOD1 mice for all three age groups. Animal number is indicated in each bar, and significant difference is indicated by asterisk $\left({ }^{*} p<0.05\right.$, $\left.{ }^{* * *} p<0.001\right)$.

The fluid rate was adjusted to $5-7 \mathrm{ml} / \mathrm{min}$, and the dura below lumbar segment was removed with eye-surgical scissors. Immediately after decapitation, the cord was transected at the L1-L2 region, and the dorsal/ ventral roots at the caudal cord were cut at their spinal outlets. The transected spinal cord with the attached roots was quickly transferred to a $100 \mathrm{~mm}$ Petri dish filled with the $\mathrm{mACSF}$ and bubbled with $95 \% \mathrm{O}_{2} / 5 \%$ $\mathrm{CO}_{2}$. After separating the ventral roots $(\mathrm{S} 1-\mathrm{S} 3)$ and the dorsal root that enters at the $\mathrm{S} 3$ segment, the cord was cut at the sacral edge of the lumber enlargement and transferred to a recording chamber. The chamber in this experiment used two arrays of bipolar electrodes for the ventral roots (S1-S3). The proximal (recording) and distal (stimulating) electrodes were separated by $5 \mathrm{~mm}$. In addition, one bipolar electrode was used to stimulate each dorsal root, one per side, which preferentially activated the $S 3$ ventral roots. All stimulating and recording electrodes were made of stainless-steel wire coated with Teflon and mounted on a plastic plate $(\sim 6 \times 7 \mathrm{~mm})$. The dorsal and ventral roots were placed on the electrodes and covered with a mineral/petroleum jelly (2:1) mixture. The chamber was perfused with ACSF composed of the following (in mM): $126 \mathrm{NaCl}, 3 \mathrm{KCl}, 1 \mathrm{NaH}_{2} \mathrm{PO}_{4}, 1.5$ $\mathrm{MgSO}_{4}, 2.5 \mathrm{CaCl}_{2}, 26 \mathrm{NaHCO}_{3}$, and 10 glucose with osmolarity adjusted to $300 \mathrm{mOsm}$ and $\mathrm{pH}$ to 7.4 by carefully adding $\mathrm{NaHCO}_{3}$. The ACSF was bubbled with $95 \% \mathrm{O}_{2} / 5 \% \mathrm{CO}_{2}$ and circulated at $2.5-3 \mathrm{ml} / \mathrm{min}$.

Ventral root recording and protocols. One root was stimulated at a time through an isolation unit (PSIU6E, Grass Instruments) that was connected to a stimulator (S88, Grass Instruments). The six recording electrodes on the proximal ventral roots were individually connected to six amplifiers (DAM 50, WPI) in differential mode with $1000 \times$ gain, high-pass filtering at $300 \mathrm{~Hz}$, and low-pass filtering at $20 \mathrm{kHz}$. The outputs of these amplifiers were transferred to an analog-to-digital interface (DT1322A, Molecular Devices) and the analog signals were digitized at $20-50 \mathrm{kHz}$ and 

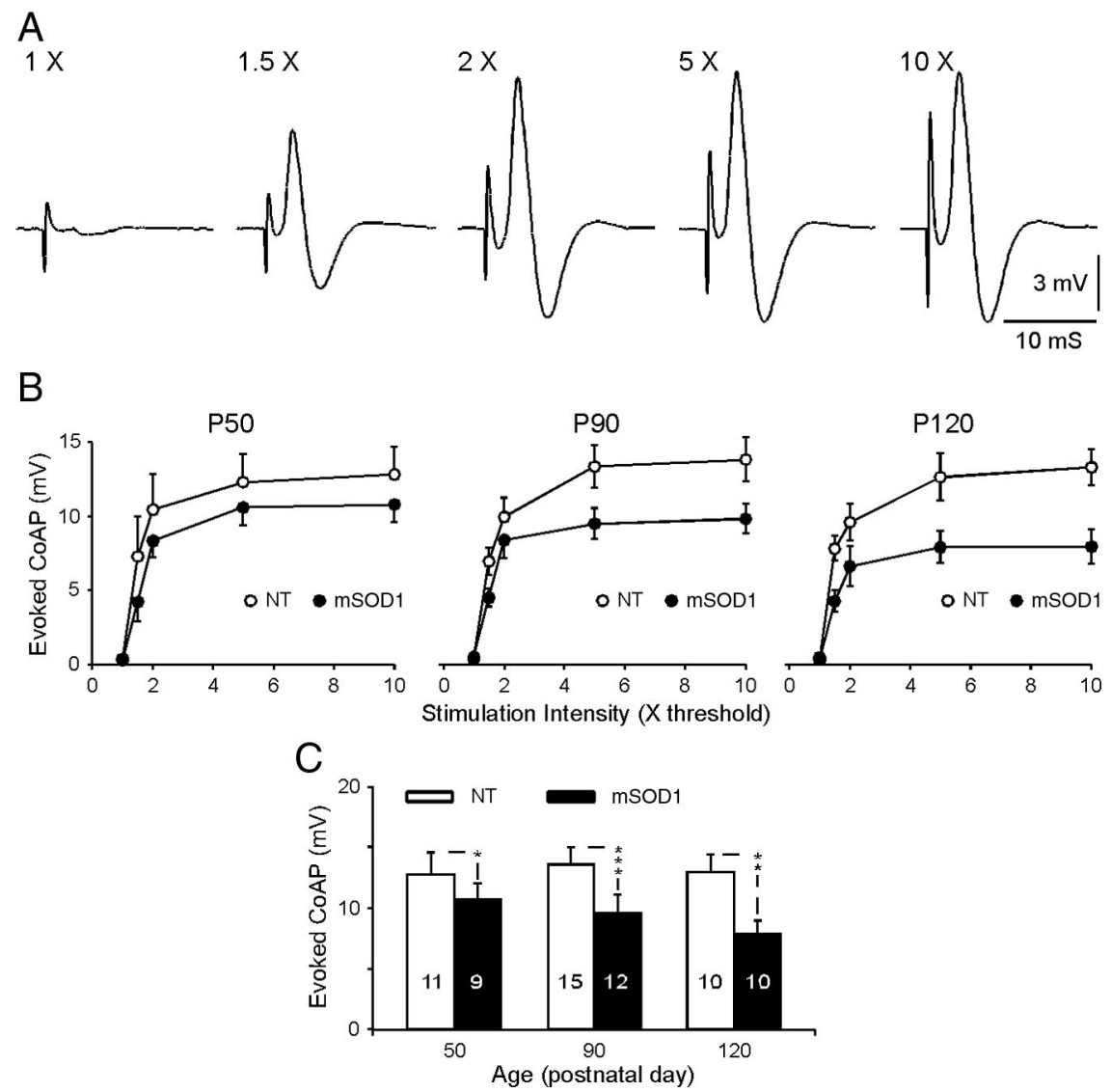

Figure 2. The antidromically activated ventral root TMA and its difference between NT and mSOD1 mice. $\boldsymbol{A}$, An example of the antidromically evoked TMA at different intensities marked as 1-10 times threshold. Note that the compound action potentials are mirrored compared with the trace in Figure 1; see Materials and Methods for explanation. $\boldsymbol{B}$, Summary plots showing the relationship between the stimulation intensity and the amplitude of the TMA in all three age groups. C, Plot of the evoked maximum TMA between NT and mSOD1 mice for three age groups. Animal number is indicated in each bar, and significant difference is indicated by asterisk $\left({ }^{*} p<0.05,{ }^{* *} p<0.01,{ }^{* * *} p<0.001\right)$.

acquired into a Dell computer controlled by pCLAMP software (version 9.1, Molecular Devices). Because the recording electrodes were of bipolar configuration, the motor output evoked from dorsal root stimulation arrived at the pole proximal to the cord first, while antidromically evoked compound APs from the distal ventral root stimulation arrived at the distal pole first. Therefore, the traces appear with opposite polarity.

The dorsal roots were stimulated with a $0.2 \mathrm{~ms}$ pulse at various intensities to evoke SRs in ventral roots. The amplitude of this evoked SR was measured from the peak-to-peak height of the initial reflex response (see Fig. 1). After the threshold of the SR was determined, the dorsal root was stimulated four times at intervals of $1 \mathrm{~min}$, and this protocol was repeated for intensities 1.5, 2, 5, and 10 times threshold. The maximum SR responses (averaged from 5 and 10 times threshold) were compared between mSOD1 and NT mice across the three age groups. To confirm the monosynaptic nature of the evoked SR, the standard deviation (SD) of the latency was calculated (Doyle and Andresen, 2001) from $>20$ trials at each stimulating intensity. Beside this SR, evoked reflexes with longer latency were rare and therefore were not included in this paper. The total motoneuron activity (TMA) was assessed by stimulation of the distal portion of the ventral roots and recording proximally. For the TMA, the stimulating pulse was set to $0.05 \mathrm{~ms}$ to reduce the stimulus artifact, and the interstimulus interval was set to $\sim 10 \mathrm{~s}$ because the evoked TMA recovered much faster than the SR. The same protocol as in the SR stimulation was used to generate the intensity-response relationship for the TMA.

Burst activity was induced by bath application of $100 \mu \mathrm{M}$ picrotoxin, a $\mathrm{GABA}_{\mathrm{A}}$ receptor antagonist, and $5 \mu \mathrm{M}$ strychnine, a glycine receptor antagonist. On a scale of seconds, as shown in Figure 4, these bursts appear to occur simultaneously in all channels, and therefore are referred to as synchronized bursts. The cycle time of the synchronized bursting for each mouse was calculated from an average of 10-20 bursts. Sub-bursts were characterized by a synchronized increase in bursting amplitude before the ongoing burst completely terminated (see Fig. 4). When the time scale was expanded to the millisecond level (see Fig. $4 \mathrm{Bb}$ ), the initiation of the bursts for each segment became clear and the burst order from the S1 to S3 segments was ranked (1, 2, 3 as the first, second, and third burst). When burst initiation between segments was within $0.2 \mathrm{~ms}$, the bursts were treated as starting at the same time and the same rank was assigned. For each segment (S1S3), a percentage was calculated based on how many times that segment was the first to burst during synchronized activity. The calculations were made from at least 10 synchronized bursts. Differences in these parameters were compared between NT and mSOD1 mice. Due to difficulty in determining the end of each burst, the burst duration was not calculated in this study.

To investigate the origination and cellular mechanism of the synchronized bursts induced by picrotoxin and strychnine, $\mathrm{Mg}^{2+}$, AMPA and kainate acid/DNQX (the agonists/ antagonist of AMPAR), and NMDA/APV (the agonist/antagonist of NMDA receptor) were used in various combinations. The characteristics of the resulting bursts were then compared between NT and mSOD1 mice.

All chemicals in this study were purchased from Sigma-Aldrich. The statistical analyses were performed with ANOVA, $\chi^{2}$ tests, Student's $t$ tests, and univariate ANOVA using SPSS software.

\section{Results}

\section{Short-latency reflexes are reduced in} mSOD1 animals as symptoms appear The changes in short-latency sensory input to spinal motoneurons in the mSOD1 mouse were evaluated by stimulating the dorsal root and recording the SR from the ventral root (Fig. 1). Because the S3 ventral root had the best response to the dorsal root stimulation in this experimental setting, only the evoked response recorded from the S3 ventral root was analyzed. The threshold for this SR was found to be 5-15 $\mu \mathrm{A}$. The stimulus-response relationship showed that the maximum SR was mostly reached at an intensity of 2 times threshold with a slight, further increase up to 5-10 times threshold intensity, as illustrated in Figure 1, $A$ and $C$. The latency for the SR was stable and short $(\sim 2 \mathrm{~ms})$ in both NT and mSOD1 mice, and therefore it is likely mediated through monosynaptic transmission, as reported by two other laboratories ( $\mathrm{Li}$ et al., 2004; Moghaddasi et al., 2007). This was further supported by jitter analysis (Doyle and Andresen, 2001), which showed little variability in the SR latency (stimulation, mean latency \pm SD: 1.5 times threshold, $2.43 \mathrm{~ms} \pm 10 \mu \mathrm{s} ; 5$ times threshold, $2.34 \mathrm{~ms} \pm 20$ $\mu$ s; $n=4$ mice). In four mice ( $2 \mathrm{NT}$ and $2 \mathrm{mSOD} 1$ ), the receptor that mediated the SR was tested. As shown in Figure 1 $B, 3-5 \mathrm{~min}$ bath application of $10 \mu \mathrm{M}$ DNQX completely blocked the SR, suggesting that this monosynaptic SR was mainly mediated through AMPAR. The averaged peak-to-peak amplitude of the SR at 5-10 times threshold was found to be significantly smaller only in the two older age groups of mSOD1 mice than NT mice (Fig. $1 D$ ) (P90: $p<0.05$; P120: $p<0.001$ ).

Motoneuron death is the major pathological consequence in ALS. For this reason, the amplitude of the SR could be reduced 
due to progressive motoneuron death instead of loss of short-latency synaptic input. To evaluate this possibility, TMA was assessed by the antidromic response at the proximal ventral root electrode to electrical stimulation of the distal ventral root. It was found that the proximal ventral root threshold was reached in response to shorter stimulation times during antidromic activation, $0.05 \mathrm{~ms}$ versus $0.2 \mathrm{~ms}$ for the SR, while the stimulus intensity required remained the same, 5-10 $\mu \mathrm{A}$. As seen in Figure 2, $A$ and $B$, the stimulusresponse relationship of the evoked TMA was similar to the SR in Figure 1. Comparison of the maximum TMA reveals that it was significantly smaller in all age groups of mSOD1 mice than that in NT mice (P50: $p<0.05$; P90: $p<0.001$; P120: $p<0.01)$, with the decrease progressing with age.

The above data showed progressive decreases in the SR and the TMA in MSOD1 mice with increased age compared with their NT littermates. Thus the decrease in the SR could be due to loss of motoneurons as reflected in the decreased TMA. If the loss of the SR was greater than the TMA, then the number of sensory synapses or their efficacy may decrease with age. Although it appears to be a steeper decline in the SR compared with TMA (compare Figs. $1 D, 2 C$ ), this was not statistically significant $(p=0.062$ by univariate ANOVA, 9-15 experiments per group). This result rules out the possibility that the $\mathrm{SR}$ is a source of increasing excitotoxic drive to motor neurons. One factor that might have influenced these results is that the mSOD1 are more prone to injury during the dissection process. The mSOD1 preparations, however, showed just as good survival times in vitro as did the NT preparations, both in terms of duration of reflex outputs and consistency of effects of dorsal root stimulation.

\section{Bursting generated by interneuronal inputs is altered after symptom onset}

A large proportion of the total synaptic input to motoneurons is mediated by spinal interneurons. Thus, a functional change in interneurons in ALS may also affect motoneuron excitability. To assess the excitability of interneuronal circuits, we analyzed the synchronized bursts recorded in ventral roots by blocking inhibitory synaptic transmission (Cowley and Schmidt, 1995; Hinckley et al., 2005). As shown in Figure $3 A$, synchronized bursts were produced in some preparations by bath application of $\mathrm{GABA}_{\mathrm{A}}$ and glycine receptor antagonists, picrotoxin $(100 \mu \mathrm{M})$ and strychnine $(5 \mu \mathrm{M})$. The synchronous coordination between roots suggests involvement of excitatory spinal interneuronal network in the burst genesis, which was supported by the blocking effects of DNQX (Fig. $3 A)(n=4)$ or APV (Fig. $3 C)(n=3)$. For the cords without the inducible bursts, the unsynchronized bursts was produced by NMDAR activation under blocking AMPAR
$100 \mu \mathrm{M}$ picrotoxin $+5 \mu \mathrm{M}$ strychnine

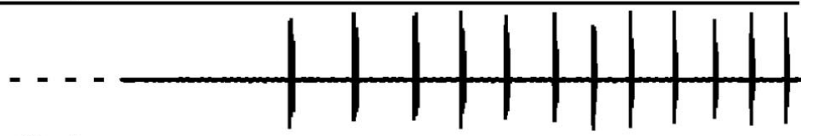

$40 \min$

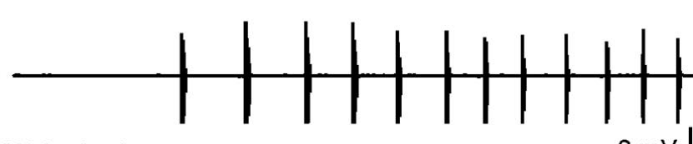

$\frac{2 \mathrm{mV}}{1 \mathrm{~min}}$

$10 \mu \mathrm{M} D N Q X$ for $3 \mathrm{~min}$

100 MMNMA

$-10-1,--------------$

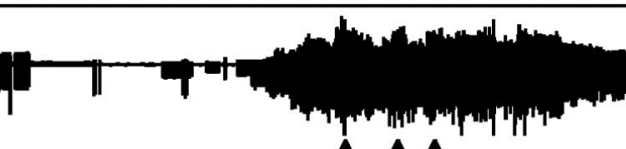

$\Delta \boldsymbol{\Delta} \Delta$

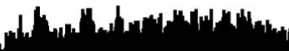

A

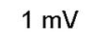

$1 \mathrm{~min}$

C $100 \mu \mathrm{M}$ picrotoxin $+5 \mu \mathrm{M}$ strychnine $100 \mu \mathrm{M}$ APV

Figure 3. Bursting activity and its mechanism. $A$, Synchronized bursts were produced by bath application of picrotoxin and strychnine. Addition of DNQX blocked the bursts, suggesting the bursts originated from premotor interneurons. $\boldsymbol{B}$, In , synchronized bursts did not occur after picrotoxin and strychnine application. Subsequent NMDA bath quent AMPA receptor activation only produced tonic firing suggesting that synchronized bursting does not originate from AMPA receptors. S1-S3 ventral roots are from the same side of the spinal cord.

(Fig. $3 B)(n=2)$, making the burst origin from strong gap junctions between motoneurons and recurrent excitation pathway unlikely in this experimental setting (see Discussion).

From the analysis of a total of 45 adult mice (in both the P90 and P120 groups), several features of these synchronized bursts were different between mSOD1 and their NT littermates. First, although not all preparations exhibited synchronized bursts following administration of picrotoxin and strychnine, the proportion of bursting preparations was higher in mSOD1 than NT (mSOD1: $21 / 26$; NT: $10 / 19 ; \chi^{2}$ test: $\chi^{2}=4.06, p<0.05$ ). The lower occurrence of bursting in NT mice could be due to selective interneuron damage during the dissection. This is, however, highly unlikely since mSOD1 cords would be more susceptible to damage due to the disease. Second, as previously reported (Cowley and Schmidt, 1995), some bursts contained multiple subbursts, which is probably due to repetitive burst initiation from spinal interneurons. As illustrated in Figure 4 and summarized in Table 1, mSOD1 mice exhibited significantly more sub-bursts than NT mice $(p<0.05)$. Third, in NT mice, most bursts started 

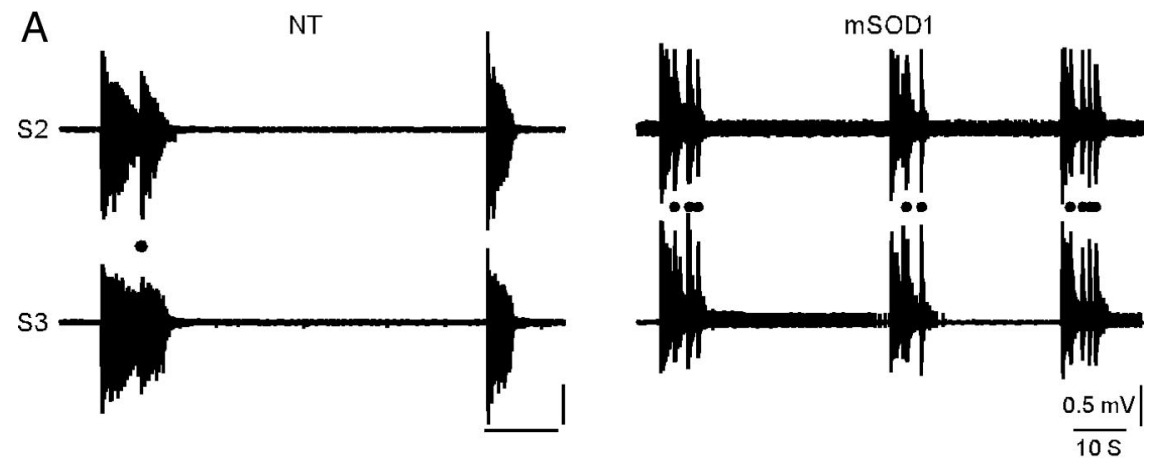

B

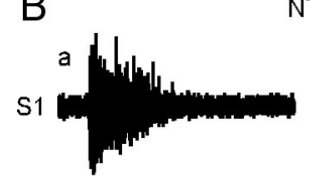

NT
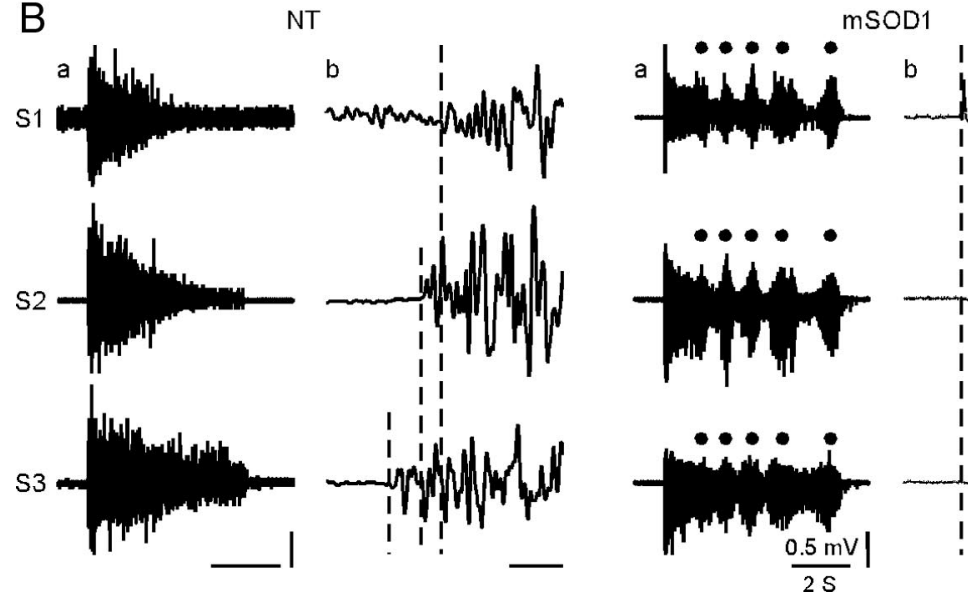

Figure 4. Characteristics of the synchronized bursts. $A$, The examples show the difference in sub-bursting architecture between NT and mSOD1 mice. These bursts are produced by bath addition of $100 \mu \mathrm{m}$ picrotoxin and $5 \mu \mathrm{m}$ strychnine in the recording solution. $\boldsymbol{B}$, The examples show initiation of the burst in S1-S3 ventral roots. Panels $\boldsymbol{a}$ show the regular displays of whole burstings including sub-bursting. When the time scale is expanded in $\boldsymbol{b}$, timing differences in burst initiation in ventral roots $\mathrm{S1}-\mathrm{S} 3$ become clear. The dashed lines indicate the starting time point. The sub-bursts are marked by the black dots. S1-S3 ventral roots are from the same side of the spinal cord.

Table 1. Occurrence of sub-bursts

\begin{tabular}{lllrr}
\hline & $\begin{array}{l}\text { Single burst } \\
\text { (mean } \pm \text { SD) }\end{array}$ & $\begin{array}{l}\text { Double bursts } \\
\text { (mean } \pm \text { SD) }\end{array}$ & $\begin{array}{l}\text { More bursts } \\
\text { (cord number) }\end{array}$ & $n$ \\
\hline NT & $76.35 \pm 21.75 \%$ & $15.41 \pm 12.88 \%$ & $3 / 7$ (yes $/$ no) & 10 \\
mS0D1 & $50.33 \pm 33.30 \%$ & $27.82 \pm 17.18 \%$ & $13 / 5$ (yes/no) & 18 \\
\hline
\end{tabular}

Statistical significance of single and double bursts was calculated by $t$ test, and the last group was calculated by $\chi^{2}$ test. $p<0.05$ for all groups.

Table 2. Distribution of burst initiation in S1-\$3 ventral roots

\begin{tabular}{lllrr}
\hline & S3 & S2 & \multicolumn{1}{c}{ S1 } & \multicolumn{1}{c}{$n$} \\
\hline NT & $87.54 \pm 14.09^{*}$ & $17.84 \pm 18.02^{\text {ns }}$ & $1.33 \pm 3.18^{* * *}$ & 8 \\
mS0D1 & $70.32 \pm 20.02^{*}$ & $22.85 \pm 23.56^{\text {ns }}$ & $29.95 \pm 19.63^{* * *}$ & 14
\end{tabular}

Data are shown as mean $\pm S D(\%)$. Statistical significance of burst initiation was calculated by $t$ test. ns, $p>0.05$; ${ }^{*} p<0.05 ;{ }^{* * *} p<0.001$.

at S3 and then quickly spread to S2 and S1. In contrast, a significantly higher portion of the synchronized bursts in $\mathrm{MSOD} 1$ mice started at S2 (Fig. $4 B b$, Table 2). In other words, the segmental order of excitability in mSOD1 interneurons is disrupted. However, the burst in mSOD1 mice does not occur at higher frequency than that in the NT mice (the averaged burst cycle, mean $\pm \mathrm{SD}$ : for NT, $1.19 \pm 0.22 \mathrm{~min}, n=9$; for $\mathrm{mSOD} 1,0.89 \pm$ $0.65 \min , n=14$; $t$ test, $p=0.2$ ).

\section{NMDARs play an important role in bursting}

It is known that the NMDAR has an important role in the generation of rhythmic bursting in the spinal cord (e.g., Schmidt et al., 1998; Bonnot et al., 2002). We tested whether this receptor contrib-

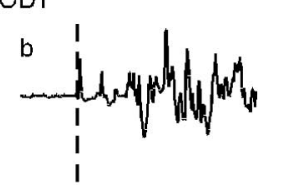

uted to the increased bursting activity generated by the interneurons in mSOD1 mice. Our data showed that administration of high doses of NMDA $(100 \mu \mathrm{M})$ following block of AMPARs by DNQX can reinitiate synchronized bursting, but at an increased frequency compared with the pre-DNQX state (Fig. 5A). The reverse experiment, blocking NMDAR and then adding AMPA, generated tonic firing but no bursting (Fig. $3 C)(n=3)$. Because AMPARs are quickly desensitized following activation by AMPA (Mayer and Vyklicky, 1989), kainate was used to activate AMPARs in four additional experiments at doses of 25,50 , and $100 \mu \mathrm{M}$. In these experiments an initial burst was followed by tonic firing, but repetitive bursting was not seen (data not shown). In the preparations that did not exhibit bursts in the presence of picrotoxin and strychnine, administration of NMDA after DNQX produced tonic activity with unsynchronized bursts (Fig. $3 B)(n=2)$. These results suggest that the NMDARs play a key role in the synchronized bursting following administration of picrotoxin and strychnine. The synchronized nature of the bursts suggests significant activation of interneurons by NMDA, though a role for NMDA acting directly on motoneurons to amplify these effects cannot be completely excluded.

We then quantified the bursts cycle mediated by administration of NMDA following DNQX, picrotoxin, and strychnine in 16 mice (NT: $n=8$, ages between P92 and P155; mSOD1: $n=8$, ages between P88 and P133). On average, the burst cycle was 9.64 s/cycle in mSOD1 mice and $10.48 \mathrm{~s} /$ cycle in NT mice. It is noticeable that initial burst cycle was shorter than the following ones in all tested mice (Fig. 5B). The reduction in the burst frequency with prolonged exposure to NMDA is likely due to receptor desensitization (Lester and Jahr, 1992; Sornarajah et al., 2008). Thus, the first bursting cycle may reflect better the intact functional state of the receptor. Statistics on the first burst cycle showed that it was significantly shorter in mSOD1 than in NT mice (NT: mean \pm SD, $8.85 \pm 2.15 \mathrm{~s}$; mSOD1: $6.50 \pm 1.9 \mathrm{~s} ; p=0.011$ ), suggesting a significant change in the excitability of NMDAR in the interneurons of mSOD1 mice.

Spontaneous synchronized bursts emerge in animals well past symptom onset

In mSOD1 mice with clear muscle tremor (in the P120 group), spontaneously occurring, synchronized bursts were detected in the absence of any drugs $(n=7)$ (Fig. 6). In general, this type of bursting was much shorter in duration than the bursts induced by picrotoxin and strychnine. It generally occurred in various combinations of two to three ventral roots. In the four cords tested, reducing $\mathrm{Mg}^{2+}$ concentration to $1 \mathrm{mM}$ (Fig. $6 \mathrm{A1}$, left) or to $0 \mathrm{~mm}$ (Fig. 6A2, control) dramatically increased bursting activity, while increasing $\mathrm{Mg}$ concentration to $2 \mathrm{mM}$ decreased it (Fig. 6A1, right). Similarly to the induced burst in Figure $3 \mathrm{~A}$, addition of 10 $\mu \mathrm{M}$ DNQX blocked the spontaneous bursts completely (Fig. $6 A 2$ ), suggesting that these bursts are driven by spontaneous activity in interneurons instead of motoneurons. In another two 
A
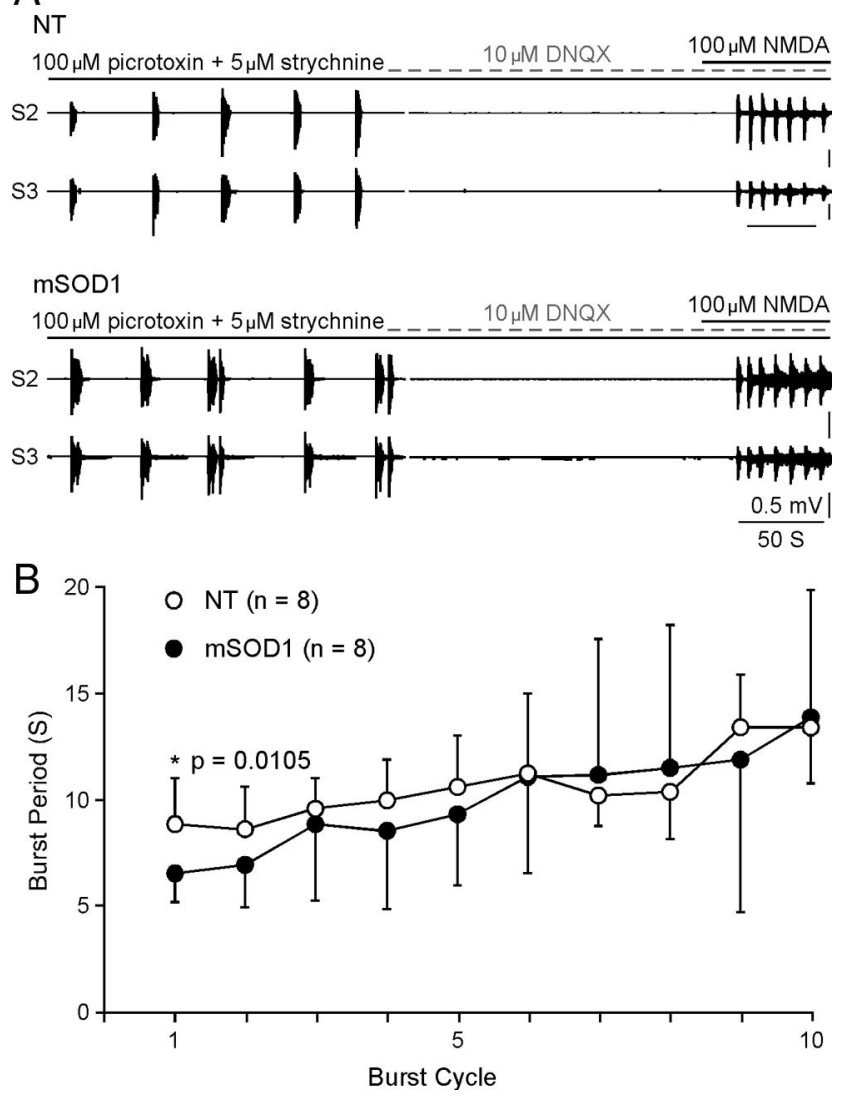

Figure 5. NMDA-induced burst. $\boldsymbol{A}$, The examples show that NMDA produced synchronized bursts in both NT (top) and mSOD1 (bottom) mice. Before NMDA application, the application of picrotoxin/strychnine and DNQX demonstrates that the synchronized burst can be generated and is coordinated by premotor interneurons. $\boldsymbol{B}$, The plot summarizes the time course of NMDAinduced bursts. Although the prolonged application of NMDA slows the bursts in both NT and mSOD1 mice, the first cycle of the burst is significantly shorter in mSOD1 mice than in NT mice. ${ }^{*} p<0.05 . S 2$ and $S 3$ ventral roots are from the same side of the spinal cord.

cords, the burst was dose-dependently inhibited by bath application of APV (Fig. $6 B$ ). These results suggest that, similar to the induced bursts, these spontaneous bursts originate in spinal interneurons and that the NMDAR is at least one contributing factor in affecting its occurrence and frequency.

\section{Discussion}

Although ALS is characterized by loss of motoneurons in the spinal cord and corticospinal neurons in the motor cortex, loss or changes in other types of neurons may occur (Geracitano et al., 2003; Spalloni et al., 2006; Stephens et al., 2006; Bories et al., 2007; van Zundert et al., 2008). Increased activity in neurons that synapse onto motoneurons could lead to increased depolarization and subsequent excitotoxicity. Our results in the mutant SOD1 mouse model of ALS indicate that short-latency, presumably monosynaptic sensory input to motoneurons, is not a source of excess excitatory input. Instead, as symptoms develop, the reflex from group I muscle afferents declines in parallel with the number of motoneurons responding to the antidromic stimulation.

In contrast, the changes in synchronous bursting patterns suggest that overall activity of spinal interneurons does change as the disease progresses. The synchronous bursting in response to block of inhibitory receptors was more common in mutant animals, suggesting an overall increase in excitability of these cells or a change in their synaptic connectivity. Pattern changes in the bursting included increased sub-bursts per burst period and disrupted ordering of bursting. It was also striking that spontaneous bursts, occurring without administration of inhibitory blockers, emerged in mSOD1 mice with clear symptomatic tremors. Blocking either AMPARs or NMDARs eliminated bursts, but only administration of NMDA following AMPAR blockade reinduced bursting patterns. Moreover, the spontaneous bursting patterns that developed as the disease became more severe were sensitive to changes in extracellular $\mathrm{Mg}^{2+}$ concentration and APV, suggesting increased activity within circuits of interneurons that either have NMDARs or project to motoneurons via NMDARs. This change may be a major source of the tremor that characterizes the later stages of the disease. Further investigations are required to determine whether these spontaneous bursts are due to upregulation in NMDARs, changes in the intrinsic properties of the interneurons, or other modifications within these circuits. Behavioral testing has shown that the inhibition of NMDARs prolonged survival in mSOD1 mice (Wang and Zhang, 2005). The present study proposes a possible cellular mechanism for that observation.

\section{Synchronous bursts are likely due to interneuronal circuits}

The locomotor pattern is generated by an interneuronal network in which the excitatory interneurons initiate the rhythmic activity and the inhibitory interneurons are responsible for alternating the motor commands (Kiehn, 2006). Thus, synchronized bursts can be produced by selectively blocking the $\mathrm{GABA}_{\mathrm{A}}$ and/or glycine receptors in these inhibitory interneurons (e.g., Cowley and Schmidt, 1995; Bonnot and Morin, 1998; Hinckley et al., 2005), which provides a way to study the excitability state in interneurons of $\mathrm{mSOD} 1$ mice in our recording setting. In these experiments, we used high concentrations of both picrotoxin and strychnine to induce reliable bursting in the adult spinal cords (González-Forero and Alvarez, 2005). Premotor excitatory interneurons are clearly required to generate these synchronous bursts, as activity was coordinated across several spinal segments and the bursts were eliminated following block of ionotropic glutamate receptors. Previous studies in neonatal preparations have demonstrated that synchronized rhythmic activity on ventral roots may be partly due to gap junctions (Tresch and Kiehn, 2000; Kiehn and Tresch, 2002) or recurrent excitatory pathway (Machacek and Hochman, 2006). Although gap junctions are found in adult spinal interneurons and motoneurons (Rash et al., 2000), their function in neuronal communication in adult animal is not well established (Chang et al., 2000). Nonetheless a role for gap junctions cannot be excluded. If recurrent excitation played a role in the synchronous burst in this study, we would expect spontaneous firing after blocking synaptic transmission with DNQX, which was rarely seen in our induced and spontaneous burst. Neonatal motoneurons also exhibit TTX-resistant oscillation in response to administration of NMDA (Hochman et al., 1994). In our experiments however, block of synaptic input resulted in block of bursting, so it is unlikely to be due to intrinsic bursting in the motoneurons.

\section{Possible mechanisms of changes in interneuronal circuits}

The basic mechanism of the synchronized bursts appears to be similar in both NT and mSOD1 mice, in which both exhibited multiple sub-bursts per overall burst. Yet the increased tendency for sub-bursts in mSOD1 animals and the other changes in burst patterns noted above suggest substantial alterations occurred within the interneuronal circuits producing bursts. In this study, we provided clear evidence showing strong involvement of 
A
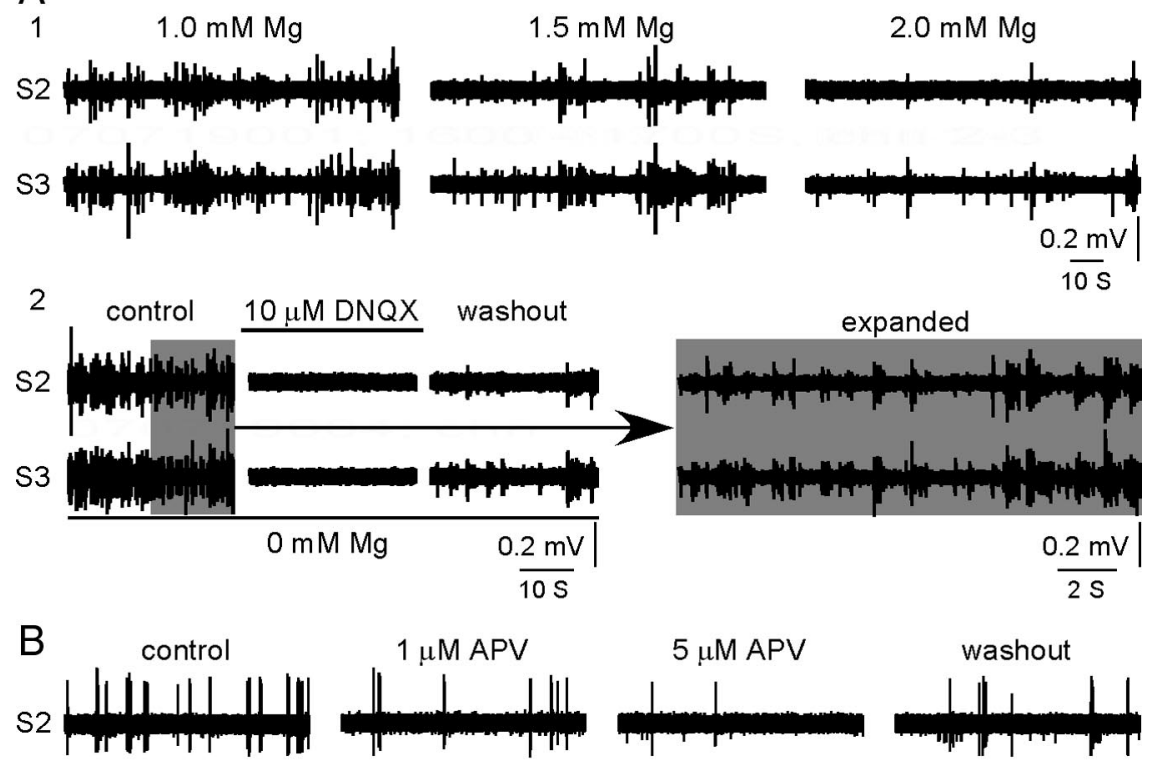

$\mathrm{S} 3$
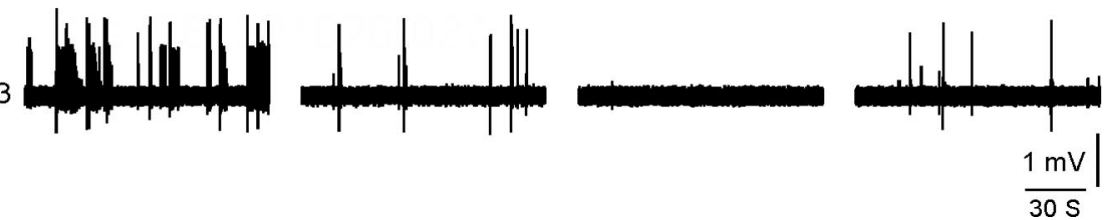

Figure 6. Spontaneous bursts in mSOD1 mice with clear tremor. $\boldsymbol{A}$, The top (1) shows the occurrence of the synchronized burst varies with the concentration of magnesium. The bottom (2) on the left was recorded under $0 \mathrm{~mm} \mathrm{Mg}^{2+}$. The burst was blocked by DNQX, indicating the burst was generated through the interneurons. The expansion on the right gives a better view of the synchronization between S2 and S3 ventral roots. $B, A$ recording shows the spontaneous and synchronized burst was dosedependently blocked by APV, the selective NMDAR antagonist. S2 and S3 ventral roots are from the same side of the spinal cord.

noted in locomotor patterns by Amendola et al. (2004), although there may be differences in this regard in sacral versus lumbar motoneurons. Thus, early deficits in motoneurons and spinal interneurons occur, likely followed by some type of adaptation mechanism. The disease progression may be caused by the compensatory mechanisms or by failure of the compensatory mechanisms. Fundamentally important questions are as follows: what are the mechanisms of compensation, and how do these mechanisms affect disease progression?

As noted above, persistent Na PIC is upregulated in mSOD1 animals at a very early age (Kuo et al., 2004, 2005; van Zundert et al., 2008). The bursting pattern from the interneurons may also involve changes in interneuronal Na PIC. Recent studies have emphasized that $\mathrm{Na}$ PICs play a major role in bursting patterns for a wide variety of spinal interneurons (Tazerart et al., 2007; Wilson et al., 2007; Zhong et al., 2007; Ziskind-Conhaim et al., 2008). If, as in motoneurons, the Na PIC is upregulated in spinal interneurons, then this change would contribute to the increased tendency for bursting, manifested in the present study as a greater percentage of preparations showing synchronous bursts after blocking inhibition and the emergence of spontaneous bursts as symptoms become intense. On the other hand, in-

NMDAR in the altered bursts in mSOD1. Thus, one important mechanism to consider is that the NMDARs themselves may be altered. Detailed studies focused on the interneuronal NMDARs are needed to determine whether receptor density, subunit composition, or its phosphorylation state are responsible for the changes shown here. The role of NMDAR does not rule out other cellular mechanisms that may contribute to the bursts recorded in mSOD1 mice. There is a loss of spinal interneurons, including their synapses and fibers to motoneurons (Schütz, 2005; Stephens et al., 2006), but it is difficult to understand how this loss would cause a greater tendency for synchronized bursts or the emergence of spontaneous bursts later in the disease. Inhibition of AMPAR has prolonged survival of mSOD1 (G93A) mice (Van Damme et al., 2003; Andries et al., 2007). Although we showed that AMPAR itself is not involved in burst generation, it may accelerate the burst by depolarizing the cell and facilitating the opening of NMDARs.

Behavior and synaptic abnormalities in early life were reported for both mutant G85R and G93A mice (Amendola et al., 2004; Bories et al., 2007; van Zundert et al., 2008). Bories et al. (2007) showed that EPSPs evoked by stimulation between 1.3 and 2.0 times threshold were reduced in these very young mutant G85R mice. In contrast, our results with G93A mice show that the reflexes produced by this input were similar in NT and mutant SOD1 mice until symptoms developed (around postnatal day 90). This age likely corresponds to the time of degeneration onset of G93A motoneuron (Schütz, 2005). While the animal strains might have differences in their synaptic responses at different ages, it is more likely that the deficits in behavior and sensory inputs recover as the animal develops and then undergo a decline as symptoms progress. A similar early deficit and then recovery was creased Na PIC in motoneurons might increase the SR, which did not occur. Changes in Na PIC over time in both motoneurons and interneurons require further study.

\section{Short-latency reflexes}

The short-latency, presumably monosynaptic reflex decreased as the disease progressed, but most of this decrease could be accounted for by loss of motoneurons (as assessed from the antidromic stimulation of the ventral root). Thus this sensory input is not a source of increased excitotoxic drive as symptoms develop. Qualitative examination of human patients with ALS has recently shown that the equivalent reflex in human subjects, the H-reflex, is markedly decreased (T. Siddique, unpublished data). The degree to which the decrease in $\mathrm{H}$-reflex also represents loss of motoneurons versus a loss in group I sensory input remains to be elucidated.

\section{Summary}

Excitotoxicity may be an important contributing mechanism causing degeneration of motoneurons in ALS. Our results indicate that NMDAR in spinal interneurons is a potential source of overexcitation of motoneurons as symptoms develop and could be a potential target for new therapeutic strategies.

\section{References}

Amendola J, Durand J (2008) Morphological differences between wild-type and transgenic superoxide dismutase 1 lumbar motoneurons in postnatal mice. J Comp Neurol 511:329-341.

Amendola J, Verrier B, Roubertoux P, Durand J (2004) Altered sensorimotor development in a transgenic mouse model of amyotrophic lateral sclerosis. Eur J Neurosci 20:2822-2826.

Andries M, Van Damme P, Robberecht W, Van Den Bosch L (2007) Iver- 
mectin inhibits AMPA receptor-mediated excitotoxicity in cultured motor neurons and extends the life span of a transgenic mouse model of amyotrophic lateral sclerosis. Neurobiol Dis 25:8-16.

Arundine M, Tymianski M (2003) Molecular mechanisms of calciumdependent neurodegeneration in excitotoxicity. Cell Calcium 34:325-337.

Bennett DJ, Gorassini M, Fouad K, Sanelli L, Han Y, Cheng J (1999) Spasticity in rats with sacral spinal cord injury. J Neurotrauma 16:69-84.

Berger ML, Veitl M, Malessa S, Sluga E, Hornykiewicz O (1992) Cholinergic markers in ALS spinal cord. J Neurol Sci 108:114-117.

Bonnot A, Morin D (1998) Hemisegmental localisation of rhythmic networks in the lumbosacral spinal cord of neonate mouse. Brain Res 793:136-148.

Bonnot A, Whelan PJ, Mentis GZ, O’Donovan MJ (2002) Locomotor-like activity generated by the neonatal mouse spinal cord. Brain Res Brain Res Rev 40:141-151.

Bories C, Amendola J, Lamotte d'Incamps B, Durand J (2007) Early electrophysiological abnormalities in lumbar motoneurons in a transgenic mouse model of amyotrophic lateral sclerosis. Eur J Neurosci 25:451-459.

Chang Q, Pereda A, Pinter MJ, Balice-Gordon RJ (2000) Nerve injury induces gap junctional coupling among axotomized adult motor neurons. J Neurosci 20:674-684.

Chiu AY, Zhai P, Dal Canto MC, Peters TM, Kwon YW, Prattis SM, Gurney ME (1995) Age-dependent penetrance of disease in a transgenic mouse model of familial amyotrophic lateral sclerosis. Mol Cell Neurosci 6:349-362.

Cleveland DW, Rothstein JD (2001) From Charcot to Lou Gehrig: deciphering selective motor neuron death in ALS. Nat Rev Neurosci 2:806-819.

Cowley KC, Schmidt BJ (1995) Effects of inhibitory amino acid antagonists on reciprocal inhibitory interactions during rhythmic motor activity in the in vitro neonatal rat spinal cord. J Neurophysiol 74:1109-1117.

Doyle MW, Andresen MC (2001) Reliability of monosynaptic sensory transmission in brain stem neurons in vitro. J Neurophysiol 85:2213-2223.

Durand J, Amendola J, Bories C, Lamotte d'Incamps B (2006) Early abnormalities in transgenic mouse models of amyotrophic lateral sclerosis. J Physiol Paris 99:211-220.

Geracitano R, Paolucci E, Prisco S, Guatteo E, Zona C, Longone P, Ammassari-Teule M, Bernardi G, Berretta N, Mercuri NB (2003) Altered long-term corticostriatal synaptic plasticity in transgenic mice overexpressing human $\mathrm{CU} / \mathrm{ZN}$ superoxide dismutase $\left(\mathrm{GLY}^{93} \rightarrow \mathrm{ALA}\right)$ mutation. Neuroscience 118:399-408.

González-Forero D, Alvarez FJ (2005) Differential postnatal maturation of GABAA, glycine receptor, and mixed synaptic currents in Renshaw cells and ventral spinal interneurons. J Neurosci 25:2010-2023.

Hamson DK, Hu JH, Krieger C, Watson NV (2002) Lumbar motoneuron fate in a mouse model of amyotrophic lateral sclerosis. Neuroreport 13:2291-2294.

Hinckley C, Seebach B, Ziskind-Conhaim L (2005) Distinct roles of glycinergic and GABAergic inhibition in coordinating locomotor-like rhythms in the neonatal mouse spinal cord. Neuroscience 131:745-758.

Hochman S, Jordan LM, Schmidt BJ (1994) TTX-resistant NMDA receptormediated voltage oscillations in mammalian lumbar motoneurons. J Neurophysiol 72:2559-2562.

Jiang MC, Heckman CJ (2006) In vitro sacral cord preparation and motoneuron recording from adult mice. J Neurosci Methods 156:31-36.

Kiehn O (2006) Locomotor circuits in the mammalian spinal cord. Annu Rev Neurosci 29:279-306.

Kiehn O, Tresch MC (2002) Gap junctions and motor behavior. Trends Neurosci 25:108-115.

Kuo JJ, Schonewille M, Siddique T, Schults AN, Fu R, Bär PR, Anelli R, Heckman CJ, Kroese AB (2004) Hyperexcitability of cultured spinal motoneurons from presymptomatic ALS mice. J Neurophysiol 91: 571-575.

Kuo JJ, Siddique T, Fu R, Heckman CJ (2005) Increased persistent $\mathrm{Na}(+)$ current and its effect on excitability in motoneurones cultured from mutant SOD1 mice. J Physiol 563:843-854.

Lester RA, Jahr CE (1992) NMDA channel behavior depends on agonist affinity. J Neurosci 12:635-643.

Li Y, Li X, Harvey PJ, Bennett DJ (2004) Effects of baclofen on spinal reflexes and persistent inward currents in motoneurons of chronic spinal rats with spasticity. J Neurophysiol 92:2694-2703.

Machacek DW, Hochman S (2006) Noradrenaline unmasks novel selfreinforcing motor circuits within the mammalian spinal cord. J Neurosci 26:5920-5928.
Mayer ML, Vyklicky L Jr (1989) Concanavalin A selectively reduces desensitization of mammalian neuronal quisqualate receptors. Proc Natl Acad Sci U S A 86:1411-1415.

Moghaddasi M, Velumian AA, Zhang L, Fehlings MG (2007) An ex vivo preparation of mature mice spinal cord to study synaptic transmission on motoneurons. J Neurosci Methods 159:1-7.

Nagao M, Misawa H, Kato S, Hirai S (1998) Loss of cholinergic synapses on the spinal motor neurons of amyotrophic lateral sclerosis. J Neuropathol Exp Neurol 57:329-333.

Pasinelli P, Brown RH (2006) Molecular biology of amyotrophic lateral sclerosis: insights from genetics. Nat Rev Neurosci 7:710-723.

Powers RK, Binder MD (2001) Input-output functions of mammalian motoneurons. Rev Physiol Biochem Pharmacol 143:137-263.

Rao SD, Weiss JH (2004) Excitotoxic and oxidative cross-talk between motor neurons and glia in ALS pathogenesis. Trends Neurosci 27:17-23.

Rash JE, Staines WA, Yasumura T, Patel D, Furman CS, Stelmack GL, Nagy JI (2000) Immunogold evidence that neuronal gap junctions in adult rat brain and spinal cord contain connexin-36 but not connexin-32 or connexin-43. Proc Natl Acad Sci U S A 97:7573-7578.

Schmidt BJ, Hochman S, MacLean JN (1998) NMDA receptor-mediated oscillatory properties: potential role in rhythm generation in the mammalian spinal cord. Ann N Y Acad Sci 860:189-202.

Schütz B (2005) Imbalanced excitatory to inhibitory synaptic input precedes motor neuron degeneration in an animal model of amyotrophic lateral sclerosis. Neurobiol Dis 20:131-140.

Sornarajah L, Vasuta OC, Zhang L, Sutton C, Li B, El-Husseini A, Raymond LA (2008) NMDA receptor desensitization regulated by direct binding to PDZ1-2 domains of PSD-95. J Neurophysiol 99:3052-3062.

Spalloni A, Geracitano R, Berretta N, Sgobio C, Bernardi G, Mercuri NB, Longone P, Ammassari-Teule M (2006) Molecular and synaptic changes in the hippocampus underlying superior spatial abilities in presymptomatic G93A+/ + mice overexpressing the human $\mathrm{Cu} / \mathrm{Zn}$ superoxide dismutase $\left(\mathrm{Gly}^{93} \rightarrow\right.$ ALA) mutation. Exp Neurol 197:505-514.

Stephens B, Guiloff RJ, Navarrete R, Newman P, Nikhar N, Lewis P (2006) Widespread loss of neuronal populations in the spinal ventral horn in sporadic motor neuron disease. A morphometric study. J Neurol Sci 244:41-58.

Tazerart S, Viemari JC, Darbon P, Vinay L, Brocard F (2007) Contribution of persistent sodium current to locomotor pattern generation in neonatal rats. J Neurophysiol 98:613-628.

Tresch MC, Kiehn O (2000) Motor coordination without action potentials in the mammalian spinal cord. Nat Neurosci 3:593-599.

Van Damme P, Leyssen M, Callewaert G, Robberecht W, Van Den Bosch L (2003) The AMPA receptor antagonist NBQX prolongs survival in a transgenic mouse model of amyotrophic lateral sclerosis. Neurosci Lett 343:81-84.

Van Den Bosch L, Van Damme P, Bogaert E, Robberecht W (2006) The role of excitotoxicity in the pathogenesis of amyotrophic lateral sclerosis. Biochim Biophys Acta 1762:1068-1082.

van Zundert B, Peuscher MH, Hynynen M, Chen A, Neve RL, Brown RH Jr, Constantine-Paton M, Bellingham MC (2008) Neonatal neuronal circuitry shows hyperexcitable disturbance in a mouse model of the adultonset neurodegenerative disease amyotrophic lateral sclerosis. J Neurosci 28:10864-10874.

Vanselow BK, Keller BU (2000) Calcium dynamics and buffering in oculomotor neurones from mouse that are particularly resistant during amyotrophic lateral sclerosis (ALS)-related motoneurone disease. J Physiol 525:433-445.

von Lewinski F, Keller BU (2005) Mitochondrial Ca2+ buffering in hypoglossal motoneurons from mouse. Neurosci Lett 380:203-208.

Wang R, Zhang D (2005) Memantine prolongs survival in an amyotrophic lateral sclerosis mouse model. Eur J Neurosci 22:2376-2380.

Wilson JM, Cowan AI, Brownstone RM (2007) Heterogeneous electrotonic coupling and synchronization of rhythmic bursting activity in mouse $\mathrm{Hb} 9$ interneurons. J Neurophysiol 98:2370-2381.

Zhong G, Masino MA, Harris-Warrick RM (2007) Persistent sodium currents participate in fictive locomotion generation in neonatal mouse spinal cord. J Neurosci 27:4507-4518.

Ziskind-Conhaim L, Wu L, Wiesner EP (2008) Persistent sodium current contributes to induced voltage oscillations in locomotor-related hb9 interneurons in the mouse spinal cord. J Neurophysiol 100:2254-2264. 